ACCEPTED MANUSCRIPT

\title{
Free evolution of an incoherent mixture of states: a quantum mechanical approach to the Van Cittert - Zernike theorem
}

To cite this article before publication: Ignacio Fabre et al 2017 Eur. J. Phys. in press https://doi.org/10.1088/1361-6404/aa8e74

\section{Manuscript version: Accepted Manuscript}

Accepted Manuscript is "the version of the article accepted for publication including all changes made as a result of the peer review process, and which may also include the addition to the article by IOP Publishing of a header, an article ID, a cover sheet and/or an 'Accepted Manuscript' watermark, but excluding any other editing, typesetting or other changes made by IOP Publishing and/or its licensors"

This Accepted Manuscript is (C) 2017 European Physical Society.

During the embargo period (the 12 month period from the publication of the Version of Record of this article), the Accepted Manuscript is fully protected by copyright and cannot be reused or reposted elsewhere.

As the Version of Record of this article is going to be / has been published on a subscription basis, this Accepted Manuscript is available for reuse under a CC BY-NC-ND 3.0 licence after the 12 month embargo period.

After the embargo period, everyone is permitted to use copy and redistribute this article for non-commercial purposes only, provided that they adhere to all the terms of the licence https://creativecommons.org/licences/by-nc-nd/3.0

Although reasonable endeavours have been taken to obtain all necessary permissions from third parties to include their copyrighted content within this article, their full citation and copyright line may not be present in this Accepted Manuscript version. Before using any content from this article, please refer to the Version of Record on IOPscience once published for full citation and copyright details, as permissions will likely be required. All third party content is fully copyright protected, unless specifically stated otherwise in the figure caption in the Version of Record.

View the article online for updates and enhancements. 


\section{Free Evolution of an Incoherent Mixture of States: A quantum mechanical approach to the Van Cittert - Zernike Theorem}

I. Fabre ${ }^{* \ddagger}$, F. Navarrete ${ }^{*} \ddagger$, L. Sarkadi ${ }^{\dagger}$ and R. O. Barrachina* $\ddagger$

* Centro Atómico Bariloche and Instituto Balseiro, Comisión Nacional de Energía Atómica (CNEA) and Universidad Nacional de Cuyo, Av. Bustillo 9500, 8400

Bariloche, Argentina.

¥ Consejo Nacional de Investigaciones Científicas y Técnicas (CONICET), Argentina.

$\dagger$ Institute for Nuclear Research of the Hungarian Academy of Sciences (MTA

Atomki), P.O. Box 51, H-4001 Debrecen, Hungary

E-mail: barra@cab.cnea.gov.ar

Abstract. We study the time evolution of an incoherent mixture of quantum states and demonstrate, in very simple terms, a quantum mechanical equivalent of van Cittert - Zernike theorem, which can be easily explained to Quantum Physics students with a basic knowledge of the density matrix theory. Finally, we exemplify this result by applying it to the quantitative analysis of the coherence of a beam of particles in atomic collisions. 
Van Cittert - Zernike theorem revisited

\section{Introduction}

The Sun is a conspicuously incoherent source of light. However, when Sunlight reaches the surface of the Earth after an 8-minute journey, it exhibits spatial coherence over a length of tens of $\mu \mathrm{m}$ (Mashaal et al 2012). The explanation of this remarkable effect, where the light emitted from an incoherent source becomes approximately coherent at large distances, relies on a theorem (Born and Wolf 1999) demonstrated by Pieter H. van Cittert in 1934 (van Cittert 1934) and, in a different context by Frits Zernike in 1938 (Zernike 1938).

Based on a direct analogy between electromagnetic and de Broglie waves, this theorem from light optics is usually applied to the study of coherent properties of particle beams (Cronin et al 2009). This strategy of borrowing results from classical optics and applying them to the study of particle's coherence, might be sound for qualitative analysis, but should be validated by a Quantum Mechanical approach for quantitative applications. For instance, an atom optics version of the van Cittert-Zernike theorem was proposed by Taylor et al (1994) in terms of a second-quantization model. More recently, Inpens and Guéry-Odelin (2010) employed a classical phase-space approach based on the Truncated Wigner equation.

Anyway, the concept that a beam of particles can gain coherence with time is far from being easy to grasp. Here we present a very simple derivation of van Cittert-Zernike theorem for an incoherent mixture of quantum states. This demonstration aims to senior undergraduate students and relies on basic concepts of Quantum Mechanics, as the density matrix theory and the Born approximation in elastic collisions, as explained in standard textbooks (See, e.g. Ballentine 2000, Bransden and Joachain 2000, Galindo and Pascual 1990, Gasiorowicz 2003, Landau and Lifshitz 1977, Schiff 1968). We hope that this approach would provide Physies students with the understanding of a fascinating but rather counterintuitive concept.

In this article we study the time evolution of an incoherent mixture of an ensemble of particles of mass $m$, describled by identical wave packets located at different positions upon a much larger region of dimension $D$. We evaluate the time evolution of the corresponding density operator $\rho$ by solving the Liouville - von Neumann equation. Actually, we demonstrate that a coherence length $\ell$ can be defined, such that for distances shorter than $\ell$ the system behaves coherently (i.e. $\rho$ can be approximated by the product of pure states). In particular, $\ell$ coincides with the coherence length of each individual wave packet for small times $t$, as expected; while for large values of $t$ the standard expression $\ell \propto \hbar t / m D$, of van Cittert - Zernike theorem is recovered. But first, let us review some basic concepts of the time evolution of an individual wave packet.

\section{Free time evolution of a wave packet}

Let us consider the time evolution of a normalized wave packet $\Phi_{t}$ for a particle of máss $m$ in the absence of a potential. For pedagogical reasons, let us assume that 


\section{Van Cittert - Zernike theorem revisited}

at time $t=0$, the wave packet $\Phi_{0}$ is separable in cartesian coordinates, namely, $\left\langle\mathbf{r} \mid \Phi_{0}\right\rangle=\left\langle x \mid \phi_{0}\right\rangle\left\langle y \mid \varphi_{0}\right\rangle\left\langle z \mid \psi_{0}\right\rangle$. Since this factorization is preserved for all times, we will only study the evolution of $\left\langle x \mid \phi_{t}\right\rangle$. However, it is important to point out that this assumption does not represent any loss of generality, since its removal is straightforward.

The calculation of the time evolution of $\phi_{t}$ is trivial in momentum representation. It reads

$$
\left\langle p \mid \phi_{t}\right\rangle=e^{-i E_{p} t / \hbar}\left\langle p \mid \phi_{0}\right\rangle
$$

with $E_{p}=p^{2} / 2 m$. The time evolution of the wave packet in coordinate space can be readily obtained,

$$
\begin{aligned}
\left\langle x \mid \phi_{t}\right\rangle & =\frac{1}{\sqrt{2 \pi \hbar}} \int_{-\infty}^{\infty} e^{i p x / \hbar}\left\langle p \mid \phi_{t}\right\rangle \mathrm{d} p \\
& =\frac{1}{\sqrt{2 \pi \hbar}} \int_{-\infty}^{\infty} e^{i\left(p x-E_{p} t\right) / \hbar}\left\langle p \mid \phi_{0}\right\rangle \mathrm{d} p
\end{aligned}
$$

It is important to note that, since the momentum distribution does not depend on time, namely $\left|\left\langle p \mid \phi_{t}\right\rangle\right|^{2}=\left|\left\langle p \mid \phi_{0}\right\rangle\right|^{2}$, all the expectation values related to $p$ are also timeindependent,

$$
\langle A(p)\rangle_{t}=\int_{-\infty}^{\infty} A(p)\left|\left\langle p \mid \phi_{t}\right\rangle\right|^{2} \mathrm{~d} p=\int_{-\infty}^{\infty} A(p)\left|\left\langle p \mid \phi_{0}\right\rangle\right|^{2} \mathrm{~d} p=\langle A(p)\rangle_{t=0},
$$

as expected for a free particle. In particular, so are $\langle p\rangle$ and $\Delta p=\sqrt{\left\langle p^{2}-\langle p\rangle^{2}\right\rangle}$. On the contrary, $\Delta x=\sqrt{\left\langle x^{2}-\langle x\rangle^{2}\right\rangle}$ varies in time as (Robinett et al 2005)

$$
(\Delta x)^{2}=(\Delta x)_{0}^{2}+2 \operatorname{cov}(x, p, 0) t / m+(\Delta p)^{2} t^{2} / m^{2},
$$

where we have defined the generalized covariance of two operators $A$ and $B$ as

$$
\operatorname{cov}(A, B, t)=\frac{1}{2}\langle[A-\langle A\rangle][B-\langle B\rangle]+[B-\langle B\rangle][A-\langle A\rangle]\rangle .
$$

\subsection{Gaussian Wave Packet}

As a working example and for pedagogical reasons, from now on we will consider a gaussian wave packet of initial full width $d$ (Kennard 1927, Darwin 1928, Lekner 2007, Cox and Lekner 2008), namely,

$$
\left\langle x \mid \phi_{0}\right\rangle=\left(\frac{2}{\pi d^{2}}\right)^{1 / 4} e^{-x^{2} / d^{2}},
$$

and

$$
\left\langle p \mid \phi_{0}\right\rangle=\left(\frac{d^{2}}{2 \pi \hbar^{2}}\right)^{1 / 4} e^{-d^{2} p^{2} / 4 \hbar^{2}} .
$$

Replacing in eqs (1) and (2), we obtain

$$
\left\langle x \mid \phi_{t}\right\rangle=\left(\frac{2 d^{2}}{\pi \sigma^{4}}\right)^{1 / 4} e^{-x^{2} / \sigma^{2}}
$$




\section{Van Cittert - Zernike theorem revisited}

and

$$
\left\langle p \mid \phi_{t}\right\rangle=\left(\frac{d^{2}}{2 \pi \hbar^{2}}\right)^{1 / 4} e^{-i E_{p} t / \hbar} e^{-d^{2} p^{2} / 4 \hbar^{2}}
$$

where we have defined

$$
\sigma=d \sqrt{1+i \frac{t}{\tau}}
$$

with $\tau=m d^{2} / 2 \hbar$.

The expectation values of $x$ and $p$ are null, $\langle x\rangle=0$ and $\langle p\rangle=0$, and $\Delta x=|\sigma|^{2} / 2 d$ and $\Delta p=\hbar / d$, which are in accordance with the general result, eq. (4), i.e. $(\Delta x)^{2}=$ $(\Delta x)_{0}^{2}+(\Delta p)^{2} t^{2} / m^{2}$, for a null $x-p$ covariance (Nicola 1972, Bradford 1976, Klein 1980). We see that the Gaussian wave function has its minimum value of $\Delta x \cdot \Delta p=\hbar / 2$ at $t=0$, and then increases (for both positive and negative times) as

$$
\Delta x \Delta p=\frac{\hbar}{2} \sqrt{1+\frac{t^{2}}{\tau^{2}}} .
$$

Let us point out that these latter results can be generalized to a wave packet of arbitrary form, whenever the characteristic time $\tau$ is redefined as $\tau \equiv m(\Delta x)_{0} / \Delta p$, as can be seen easily seen in eq. (4).

Finally, the Gaussian wave functions in space (8) and momentum representations can be related by this simple equation, $\left\langle x \mid \phi_{t}\right\rangle=\sqrt{2 \hbar / \sigma^{2}}\left\langle p \mid \phi_{t}\right\rangle$ with $p=2 \hbar x / \sigma^{2} ;$ which, for $t \gg \tau$, reads

$$
\left\langle x \mid \phi_{t}\right\rangle \approx\left(\frac{m}{i t}\right)^{1 / 2} e^{i m x^{2} / 2 \hbar t}\left\langle p \mid \phi_{0}\right\rangle \quad \text { with } \quad p=\frac{m x}{t}
$$

It can be easily shown, by means of a stationary phase approximation of (2), that this asymptotic limit is valid for any functional form of $\phi_{0}$ (Dollard 1971), and not only for Gaussian wave functions. We will return to this general result in section 4 .

\subsection{Coherence Length}

When a compressed wave packet is colliding with a structured target, the result would strongly depend on the distance between the source of the projectiles and the target region. The reason is that if this distance is too short, then the wave packet might not illuminate the full target coherently, but only a portion of it. But as time goes by, the width of the wave packet will increase, until it reaches a critical distance, where all the target will be illuminated coherently.

To be more specific, let us consider that the wave interacts with two similar delta potentials separated by a given distance $x_{o}$, namely $V_{+}(x) \propto \delta\left(x-x_{o} / 2\right)$ and $V_{-}(x) \propto \delta\left(x+x_{o} / 2\right)$. In a first Born approximation (See, e.g. Bransden and Joachain 2000, Galindo and Pascual 1990, Gasiorowicz 2003, Landau and Lifshitz 1977, Schiff 1968), the cross section, proportional to the squared modulus of the transition matrix element $\left\langle p\left|V_{+}+V_{-}\right| \phi_{t}\right\rangle$, would present an interference term given by

$$
I(p)=2 \operatorname{Re}\left\langle p\left|V_{-}\right| \phi_{t}\right\rangle\left\langle\phi_{t}\left|V_{+}\right| p\right\rangle \propto \exp \left(-\frac{x_{o}^{2}}{2 \ell^{2}}\right) \cos \left(p x_{o} / \hbar\right)
$$


Van Cittert - Zernike theorem revisited

with

$$
\ell=2 \Delta x=d \sqrt{1+\frac{t^{2}}{\tau^{2}}}
$$

This term would be sizable for targets of the order of $x_{o}=\ell$ or smaller. Thus, we might define $\ell$ as the "coherence" length of the wave function at a given time $t$. This definition, which relies on the conditions necessary for the appearance of an interference structure, is standard in optical studies (Svelto 2010), but still might differ from other diverse and even contradictory definitions. For instance, Cohen-Tannoudji and Guéry-Odelin (2011) define the coherence length as the width of the global spatial coherence,

$$
G\left(x_{o}\right)=\int_{-\infty}^{\infty}\left\langle x-x_{o} / 2 \mid \phi_{t}\right\rangle\left\langle\phi \mid x+x_{o} / 2\right\rangle \mathrm{d} x,
$$

corresponding to the overlapping between two wave packets separated by a distance $x_{o}$. For a gaussian wave packet (eqs. (8) and (9)) we obtain, $G\left(x_{o}\right)=e^{-x_{o}^{2} / 2 d^{2}}$. Thus, in the approach by Cohen-Tannoudji and Guéry-Odelin (2011), the coherence length is time-independent and equal to $d$.

Here, on the contrary, we retain the definition (14). Note that the more sharply peaked the wave packet is at $t=0$ (i.e. for smaller values of $d$ ), the faster its coherence length spreads (for $t>0$ ) since

$$
\ell \approx \frac{2 \hbar t}{m d} \quad \text { for }
$$

As a side comment, let us point out that in Optics this limit is named in honor of Joseph von Fraunhofer, although he was not/actually involved in the development of the theory. Putting some numbers; let us consider an electron impulsively removed from the ground state of a Hydrogen atom. For a corresponding initial width of $d=1 \AA$, the time necessary to achieve condition (16) is in the range of tens of attoseconds (i.e. $\tau=40 \mathrm{as}$ ), whereas the coherence length increases at a constant rate, $\ell / t \approx 23 \mathrm{~km} / \mathrm{s}$.

Finally, let us assume that the wave has a group velocity $\mathbf{v}$, such that it travels a distance $\mathbf{z}=\mathbf{v} t$ in a given time $t$. Even thought we are considering a one dimensional problem, $\mathbf{v}$ might not be necessarily parallel to the direction $\hat{\mathbf{x}}$. We will come back to this point in the following section. Now, replacing $\mathbf{z}=\mathbf{v} t$ in the previous equation, we obtain,

$$
\ell \approx \frac{\lambda}{\pi d} z \quad \text { for } \quad \ell \gg d,
$$

with $\lambda=h / m v$ the particle's wavelength.

\section{Time evolution of an incoherent mixture of wave packets}

Let us now consider the free evolution of an incoherent mixture of an ensemble of particles described by wave packets $\left\langle x \mid \phi_{t}\right\rangle$, as defined in (2), which are identical except for their initial positions $b$, which are distributed along the $x$ axis according to a 
Van Cittert - Zernike theorem revisited

normalized distribution $f(b)$. Initially (i.e. at $t=0$ ), the corresponding density operator $\rho(t)$ reads,

$$
\left\langle x|\rho(0)| x^{\prime}\right\rangle=\int \mathrm{d} b f(b)\left\langle x-b \mid \phi_{0}\right\rangle\left\langle\phi_{0} \mid x^{\prime}-b\right\rangle,
$$

in space representation, and $\left\langle p|\rho(0)| p^{\prime}\right\rangle=\left\langle p \mid \phi_{0}\right\rangle\left\langle\phi_{0} \mid p^{\prime}\right\rangle \tilde{f}\left(p-p^{\prime}\right)$ in momentum representation, with $\tilde{f}(q)=\int \mathrm{d} b e^{-i q \cdot b} f(b)$.

As in the previous sections, and for the sake of simplicity, we are considering a onedimensional case. This does not mean any loss of generality, since the generalization to the three dimensional case is straightforward. The density operator $\rho(t)$ evolves in time according to the the Liouville - von Neumann equation with a free Hamiltonian $H_{o}$,

$$
\frac{\partial \rho}{\partial t}=\frac{i}{\hbar}\left[\rho, H_{o}\right] \text {. }
$$

This equation is trivially solved in momentum representation, namely

$$
\left\langle p|\rho(t)| p^{\prime}\right\rangle=\left\langle p|\rho(0)| p^{\prime}\right\rangle e^{-i\left(E_{p}-E_{p^{\prime}}\right) t / \hbar}
$$

with $E_{p}=p^{2} / 2 m$; from which the time dependent expression for the space dependent matrix element can be easily obtained,

$$
\left\langle x|\rho(t)| x^{\prime}\right\rangle=\frac{1}{2 \pi \hbar} \int \mathrm{d} p \mathrm{~d} p^{\prime}\left\langle p|\rho(t)| p^{\prime}\right\rangle e^{i\left(p x-p^{\prime} x^{\prime}\right) / \hbar} .
$$

\subsection{Gaussian wave packet and impact parameter distribution}

In what follows, we will consider that both the wave packets $\phi$ and the distribution $f(b)$ are of a gaussian shape, namely,

$$
\left\langle x \mid \phi_{0}\right\rangle=\left(\frac{2}{\pi d^{2}}\right)^{1 / 4} e^{-x^{2} / d^{2}}
$$

and

$$
f(b)=\left(\frac{2}{\pi D^{2}}\right)^{1 / 2} e^{-2 b^{2} / D^{2}} .
$$

Thus, in momentum representation,

$$
\left\langle p|\rho(t)| p^{\prime}\right\rangle=\left\langle p \mid \phi_{t}\right\rangle\left\langle\phi_{t} \mid p^{\prime}\right\rangle e^{-D^{2}\left(p-p^{\prime}\right)^{2} / 8 \hbar^{2}},
$$

with $\left\langle p \mid \phi_{t}\right\rangle$ given by (9), namely,

$$
\left\langle p \mid \phi_{t}\right\rangle=\left(\frac{d^{2}}{2 \pi \hbar^{2}}\right)^{1 / 4} e^{-i E_{p} t / \hbar} e^{-d^{2} p^{2} / 4 \hbar^{2}} .
$$

Similarly, in space representation,

$$
\begin{aligned}
& \left\langle x|\rho(t)| x^{\prime}\right\rangle=\left\langle x \mid \phi_{t}\right\rangle\left\langle\phi_{t} \mid x^{\prime}\right\rangle e^{-\left(x-x^{\prime}\right)^{2} / 2 \Sigma^{2}}, \\
& \Sigma=\frac{d}{D} \sqrt{D^{2}+d^{2}+d^{2} t^{2} / \tau^{2}}
\end{aligned}
$$


with $\tau=m d^{2} / 2 \hbar$, and

$$
\left\langle x \mid \phi_{t}\right\rangle=\left(\frac{2 \operatorname{Re}\left(\sigma^{2}\right)}{\pi \sigma^{4}}\right)^{1 / 4} e^{-x^{2} / \sigma^{2}}
$$

with

$$
\sigma=\sqrt{\frac{D^{2}+d^{2}+d^{2} t^{2} / \tau^{2}}{1-i t / \tau}}
$$

Let us note that in both space and momentum representations, the density matrix (26) is composed of a gaussian pure state, incoherently modulated by a gaussian distribution of standard deviation $\Sigma$. We will come back to this result in the following section. But first, let us notice that, as expected,

$$
\operatorname{Tr}(\rho)=\int_{-\infty}^{\infty}\langle x|\rho| x\rangle \mathrm{d} x=\int_{-\infty}^{\infty}\left|\left\langle x \mid \phi_{t}\right\rangle\right|^{2} \mathrm{~d} x=1
$$

while

$$
\operatorname{Tr}\left(\rho^{2}\right)=\left(1+\frac{D^{2}}{d^{2}}\right)^{-1 / 2}
$$

so that the total coherence of the beam remains constant in time. Let us point out that a simple calculation in momentum space can show that $\operatorname{Tr}\left(\rho^{2}\right)$ is independent of time, independently of the functional forms employed for $\phi_{0}$ and $f(b)$.

\subsection{Emerging coherence from an incoherent mixture of wave packets}

It is clear that for $D \rightarrow 0$, the width $\Sigma$ of the modulating gaussian reaches infinity, and the system becomes completely coherent, consisting of a single gaussian wave centered at $x=0$. The corresponding density matrix element reads

$$
\left\langle x|\rho(t)| x^{\prime}\right\rangle=\left\langle x \mid \phi_{t}\right\rangle\left\langle\phi_{t} \mid x^{\prime}\right\rangle
$$

with a wave function given by (8), i.e.

$$
\left\langle x \mid \phi_{t}\right\rangle=\left(\frac{2 d^{2}}{\pi \sigma^{4}}\right)^{1 / 4} e^{-x^{2} / \sigma^{2}}
$$

where

$$
\sigma=d \sqrt{1+i \frac{t}{\tau}}
$$

Without going all the way to this purely coherent case, we see that for any finite value of $\Sigma$ it would be possible to define a characteristic distance for which, if $\left|x^{\prime}-x\right|$ is smaller than it, the modulating gaussian would be approximately constant and the system would mimic a completely coherent state. The emergence of this spatial coherence out of an incoherent system is the essence of the van Cittert - Zernike theorem. 7 Now, we find the same dependence on $x_{o}$ for the interference term (13) than the one obtained in the previous section, i.e.

$$
I(p)=2 \operatorname{Re}\left\langle p\left|V_{-} \rho V_{+}\right| p\right\rangle \propto \exp \left(-\frac{x_{o}^{2}}{2 \ell^{2}}\right) \cos \left(p x_{o} / \hbar\right),
$$


Van Cittert - Zernike theorem revisited

but with a different coherence length,

$$
\ell=\sqrt{d^{2}+\frac{4 \hbar^{2} t^{2}}{m^{2}\left(d^{2}+D^{2}\right)}} .
$$

Note that for $D=0$, we recover the same result obtained in the previous section for a purely coherent system.

As a marginal note, let us point out that, when dealing with the concept of spatial coherence, the corresponding coherence length $\ell$ might be defined as the distance between two points in space for which the right term in (35) is a given fraction $\xi$ of its maximum value. Thus, in general, we might define

$$
\ell=\sqrt{|2 \ln \xi|} \sqrt{d^{2}+\frac{4 \hbar^{2} t^{2}}{m^{2}\left(d^{2}+D^{2}\right)}} .
$$

A standard definition is that of the full-width at half-maximum (FWHM), for which $\xi=1 / 2$. In our case, we have arbitrarily chosen $\xi=e^{-1 / 2}$, so that (36) is obtained.

\subsection{Small and large $t$ limits}

For very small values of $t$ (a limit that in Optics is usually named after Augustin-Jean Fresnel), we obtain $\ell \approx d$. This means that the coherence length is limited to that of an individual wave packet. On the contrary, for $t \gg \tau \sqrt{1+D^{2} / d^{2}}$, it grows linearly with time as

$$
\ell \approx \frac{2 \hbar t}{m \sqrt{d^{2}+D^{2}}}
$$

Let us also point out that in general situations, $d \ll D$. This is the case, for instance, in particle accelerators, where the projectile's wave packets are much smaller than the macroscopic size of the collimators. In these cases, for $t \gg m D d / 2 \hbar$, we can approximate (36) by,

$$
\ell \approx \frac{2 \hbar t}{m D} \text {. }
$$

Finally, replacing $t=z / v$, we write equations (36) and (39) as,

$$
\ell=\sqrt{d^{2}+\left(\frac{\lambda z}{\pi \sqrt{d^{2}+D^{2}}}\right)^{2}},
$$

and

$$
\ell \approx \frac{\lambda}{\pi D} z
$$

respectively, with $\lambda=h / m v$ the particle's wavelength. As we already mentioned in a previous section, the velocity $\mathbf{v}$ is not necessarily parallel to $x$. For instance, in the standard geometry of the collision experiments, the beam of particles is moving in a direction perpendicular to that of the impact parameter $\mathbf{b}$, a situation that is usually referred to as of "transversal" coherence. 
Finally, let us point out that by comparing equations (14) and (36), we see that the coherence length grows at a much faster rate for a coherent state than in an incoherent superposition. In particular, for large values of $t$, the corresponding ratio becomes proportional to $\sqrt{1+D^{2} / d^{2}}$. This characteristic of the coherence length evolution is already evident when comparing the generalized widths $\sigma$ in equations (8) and (26).

\section{Non-gaussian wave functions and position distribution}

In the previous sections, we have assumed Gaussian shapes for the initial position distribution $f(b)$ and the wave packets $\phi$. The reason for this choice was purely pedagogical, since all the calculations can be performed analytically. In this respect let us point out that this analysis in terms of Gaussian functions is simple enough as to be included in a problem to be solved by the student. However, it is important to point out that most of the results obtained with Gaussian functions are also valid for general non-gaussian $f(b)$ and $\phi$ functions. Take equation (39), for instance; one of the main results associated to the van Cittert-Zernike theorem. By replacing the general asymptotic form (12) of the wave function in the interference term (13), a simple demonstration leads to $\ell \propto t / d$ for pure states. Similarly, replacing (12) in (35), and performing a straightforward change of the integration variable, it can be shown that for an incoherent mixture of wave functions,

$$
\ell \propto \frac{t}{D}
$$

for large values of $t$. Perhaps, this latter demonstration is too cumbersome to be of any pedagogical value. However, it can be numerically tested for different functional forms of $f(b)$ and $\phi$, providing a complementary avenue for exploration and/or exercises.

\section{Conclusions}

We have shown in section 3 that it is possible to develop a generalization of van Cittert - Zernike theorem for massive particles, as a direct application of the density matrix concept. In particular, we see that this analysis does not imply any difficulty greater than those encountered in the study of the coherence length of a pure state, as shown in section 2. Furthermore, similar results are obtained in both cases, but with the difference that for large values of $t$ the characteristic distance for the incoherent mixture is the size $D$ of the source, instead of the initial width $d$ of each of its individual waves. The reason for this result is that coherence emerges as result of the superposition of each of the indiyidual components of the mixture. In spite of its simplicity, this difference is trascendental, and goes to the very heart of the van Cittert - Zernike theorem. It makes the coherence length of the Sunlight to be of the order of tens of $\mu \mathrm{m}$, instead of larger than the Earth itself, as it would be for each of its individual photons. The same occurs in a particle accelerator, where the coherence length at the target region is of atomic dimensions and can be varied by changing its relative position along the beam's line. 
Recently, this peculiarity of the van Cittert - Zernike theorem has made it possible to develop a new set of atomic and molecular collision experiments where the cross sections are shown to depend on the degree of coherence of the projectiles beam. The coherence properties of particle beams have been extensively studied for decades, most notably in connection with neutrino interference and oscillations (Nussinov 1976, Kayser 1981), but represents a rather new issue in the area of atomic collisions (Egodapitiya et al 2011). It has been usually assumed that under very general and common conditions, the outcome of a scattering experiment does not depend on the properties of the projectiles beam (Taylor 1972). However, recent evidence in ionization (Egodapitiya et al 2011) and electron capture (Sharma et al 2012) experiments points to a breakdown of these conditions and a dependence of the collision outcome on the incident beams coherence properties. Finally let us point out that in the present article we have focussed our attention on the dependence of the spatial degree of coherence with the distance to the source, but not on its intensity distribution. This element of van Cittert Zernike theorem is relevant in Optics, but not in its application to atomic collision experiments, where the projectile's beam is assumed to be homogeneously distributed over the transversal plane.

\section{Acknowledgments}

This work was supported by Universidad Nacional de Cuyo (Grants 06/C480 and 06/C487) and by the National Scientific Research Foundation (OTKA, Grant No. $\mathrm{K} 109440)$.

\section{References}

Ballentine L E 2000 Quantum Mechanics, A Modern Development (World Scientific, Singapore) ch. 2 Born M and Wolf E 1999 Principles of Optics 7th expanded Ed. (Cambridge University Press) ch. 10 Bradford H M 1976 Am. J. Phys. 441058

Bransden B H and Joachain C J 2000 Quantum Mechanics 2nd edition (Pearson Education Ltd, Harlow) ch. 14

Cohen-Tannoudji C/and Guéry-Odelin D 2011 Advances in Atomic Physics (World Scientific) p 411

Cox C and Lekner J 2008 Eur. J. Phys. 29671

Cronin A D, Schmiedmayer J and Pritchard D E 2009 Rev. Mod. Phys. 811051

Darwin C G 1928 Proc. R. Soc. A 117

Dollard J D 1971 Rocky Mountain J. Math. 15

Egodapitiya K N, Sharma S, Hasan A, Laforge A C, Madison D H, Moshammer R and Schulz M 2011 Phys. Rev. Lett. 106153202

Galindo A and Pascual P 1990 Quantum Mechanics I (Springer-Verlag, Berlin) ch. 2

Gasiorowicz S 2003 it Quantum Physics 3rd edition (John Wiley, Hoboken) Suppl. 20-c

Impens F and Guéry-Odelin D 2010 Phys. Rev. A 81065602

Kayser B 1981 Phys. Rev. D 24110

Kennard E H 1927 Z. Phys. 44326

Klein J R 1980 Am. J. Phys. 481035

Landau L D and Lifshitz E M 1977 Quantum Mechanics, Non relativistic Theory 3rd. edition (Pergamon Press, Oxford) ch. 2 


\section{Van Cittert-Zernike theorem revisited}

Lekner J 2007 Am. J. Phys. 751151

Mashaal H, Goldstein A, Feuermann D and Gordon J M 2012 Opt. Lett. 373516

Nicola M 1972 Am. J. Phys. 40342

Nussinov S 1976 Phys. Lett. B 63201

Robinett R W, Doncheski M A, Bassett L C 2005 Found. Phys. Lett. 18455

Schiff L I 1968 Quantum Mechanics 3rd edition (McGraw-Hill, New York) ch. 10 Sharma S et al. 2012 Phys. Rev. A 86022706

Svelto O 2010 Principles of Lasers, 5th edition (Springer)

Taylor J R 1972 Scattering Theory: The Quantum Theory on Nonrelativistic Collisions (John Wiley \& Sons Inc, Hoboken, New Jersey)

Taylor B, Schernthanner K J, Lenz G and Meystre P 1984 Opt. Comm. 110569 van Cittert P H 1934 Physica 1201

Zernike F 1938 Physica 5785 